\title{
Design of a Multiorder OFDM Frequency Diversity Approach
}

\author{
Abdeldime Mohamed Salih Abdelgader ${ }^{1,2}$ and Lenan $\mathrm{Wu}^{1}$ \\ ${ }^{1}$ School of Information Science \& Engineering, Southeast University, Nanjing 210096, China \\ ${ }^{2}$ Karary University, 12304 Khartoum, Sudan \\ Correspondence should be addressed to Abdeldime Mohamed Salih Abdelgader; abdeldime@hotmail.com \\ Received 14 September 2014; Revised 22 January 2015; Accepted 29 January 2015 \\ Academic Editor: Hsuan-Ling Kao
}

Copyright (C) 2015 A. M. S. Abdelgader and L. Wu. This is an open access article distributed under the Creative Commons Attribution License, which permits unrestricted use, distribution, and reproduction in any medium, provided the original work is properly cited.

\begin{abstract}
Frequency diversity is used to reduce the effect of destructive fading, so as to improve the communication quality, by passing the information symbols through multiple independently faded paths, and ensure that reliable communication is possible as long as one of these paths is strong. In this paper a multiorder orthogonal frequency division multiplexing (OFDM) frequency diversity approach using properties of order theory and Hamming distance is proposed. The frequency diversity is obtained by specifying proper correlations among the transmitted symbols. As subchannels experience independent fading, at least one of the symbols may have robust signal, which can be used by the receiver to detect other symbols. Considering bit error rate (BER) performance, power consumption, bandwidth utilization, and practical implementation expense, simulation results show that the proposed approach outperforms other OFDM diversity techniques, such as Maximal Ratio Combination (MRC) and Space Frequency Block Coding (SFBC).
\end{abstract}

\section{Introduction}

The recent development in data transmission necessitates dependable wireless communication systems, which are required to function at multilevel of transmission rate. The major handicaps of wireless communications systems are interference and fading caused by destructive addition of multipath in the channel $[1,2]$. Diversity techniques are widely involved in the recent communication systems owing to their significant contribution in mitigating fading effects. Diversity provides the receiver with many independent replicas of the transmitted signal. Therefore, it is a powerful technique to increase immunity of the communication system against both fading and interference [3]. Common forms of diversity are frequency diversity, time diversity $[1,4-6]$, and spatial diversity [7].

Orthogonal frequency division multiplexing (OFDM) is a multicarrier system that can simultaneously transmit symbols in several subchannels [8]. In OFDM systems, the period of the transmitted symbols significantly increases compared to that of single carrier systems, so that the immunity against the effect of multipath considerably increases.
System reliability can be enhanced using diversity techniques, without increasing the transmit power or spreading channel band. Some forms of diversity schemes such as Maximal Ratio Combination (MRC-OFDM) in $[9,10]$ can gain frequency diversity by transmitting the same symbol in several subchannels and combining the received data by applying amplitude weighted techniques and phase compensation. In spite of that, the efficiency of system is reduced because the same symbol is repetitively transmitted. Space-Frequency Block Coding (SFBC-OFDM) schemes in $[6,11-14]$ employ spacetime codes across OFDM symbols and transmit symbols in both space and frequency domains. The weaknesses of these techniques are their relatively high cost and complexity of practical implementation, because they necessitate the use of multiantennas.

Currently, a lattice signal form has been used in singleantenna single-carrier system's time diversity technology under time varying channel [15]. Besides, some space-time code in multiantenna system has also been designed based on lattice signals to attain space diversity [16]. The time diversity is obtained in [15] by using mutual independence channel fading at several time-slots in time varying channel, 
while space diversity is attained in [16] by exploiting mutual independence spatial fading in MIMO system.

In this paper, a new multiorder OFDM frequency diversity approach is proposed to improve the wireless communication system performance, without reducing efficiency or increasing implementation cost. The proposed approach utilizes the multisymbols joint design based on order theory, mainly algebraic lattice, and the Hamming distances of the transmitted symbol vectors. Considering the traditional characteristic of the OFDM, the generated symbols are transmitted in different and independent subcarriers in such a way that a frequency diversity gain can be obtained. The diversity gain is achieved by utilizing algebraic lattice properties to create a kind of relation between the transmitted symbols.

In this paper, a brief revision about OFDM and previous related frequency diversity techniques is presented in Section 2. In Section 3, the proposed approach is presented and extensively analysed. Simulation settings and results are presented in Section 4. Section 5 concludes this work.

\section{OFDM and Diversity}

2.1. OFDM Principles. OFDM is a multicarrier transmission technique that divides the available spectrum $(N)$ into many subcarriers $(F)$; each one is modulated by a low rate data stream [17]. OFDM efficiently utilizes the available spectrum by using orthogonal subcarriers. Orthogonality of the subcarriers makes the spectrum of each carrier has a null at the centre of each other; thus, carriers can be spaced very close without intercarrier interference (ICI). OFDM is capable of converting frequency selective channels into several independent flat fading subchannels; subsequently it greatly mitigates the effect of multipath $[1,3,8,9,18]$.

OFDM principles assume that there are $N$ orthogonal basis functions or pulse shapes $g(t)$, with $k=0,1,2, \ldots, N$. In the $n$th symbol interval $N$ symbols $a[n]$ from a certain symbol alphabet are transmitted simultaneously. These symbols are amplitude factors of $N$ orthogonal pulses $g_{k}\left(t-n T_{s}\right)$. Thus the transmitted signal component corresponding to the $n$th symbol interval is as in (1), while the overall transmitted symbols without the guard interval can be expressed by (2):

$$
\begin{gathered}
s_{n}(t)=\sum_{k=0}^{N-1} a_{k}[n] g_{k}\left(t-n T_{s}\right) e^{j 2 \pi k F t}, \\
s(t)=\sum_{-\infty}^{\infty} \sum_{k=0}^{N-1} a_{k}[n] g_{k}\left(t-n T_{s}\right) e^{j 2 \pi k F t} .
\end{gathered}
$$

For the easiest practical digital implementation a discrete version of the above signal using sampling can be

$$
s[m]=\frac{1}{\sqrt{N}} \sum_{n=-\infty}^{\infty} \sum_{k=0}^{N-1} a_{k}[n] e^{j 2 \pi(k m / N)},
$$

where $m$ here is the discrete time variable.

This signal will pass through a noisy multipath fading channel, modelled by FIR $h(\tau)$ of length $\tau_{\max }$, yielding the

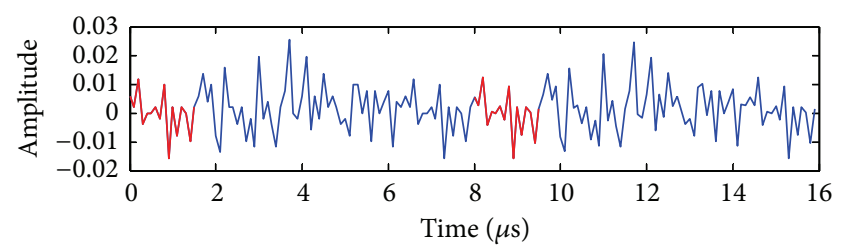

FIGURE 1: Simple waveform representation of two OFDM symbols with their corresponding (GI).

length $L$ discrete time impulse response $h[l]$, and additive white noise $w(0, \delta)$; the received signal can be expressed as

$$
r[m]=\sum_{l=0}^{L-1} h[l] s[m-l]+w(m) .
$$

ISI can be avoided by adding the guard interval (GI) between the successive symbols with a length not less than $L$, which is removed before the FFT process in the receiver [19]. Time domain waveform representation of two OFDM symbols with their corresponding (GI) is shown in Figure 1.

2.2. Maximal Ratio Combining OFDM. MRC-OFDM as one of frequency diversity techniques is a means of combining the signals from all receive paths, so that signals with a higher received power have a larger influence on the final output, which improve SNR [9]. All signal paths are cophased and summed with optimal weighting to maximize combiner output SNR. After transmission through $U$ independent subchannels with corresponding channel gain $\alpha_{u}, u=1, \ldots, U$, the modulated OFDM signal $x(t)$ can be received as

$$
r_{u}(t)=\alpha_{u}(t) e^{j \theta_{u}(t)} x(t)+W_{u}(t),
$$

where $\alpha_{u}(t)$ and $W_{u}(t)$ are, respectively, the channel gain and additive Gaussian noise, in the $u$ th subchannel. In the receiver, it can be combined using $\alpha_{u}$ as weighted factors to get the summed signal. The output SNR is the sum of all the input SNR [19]:

$$
\gamma_{\mathrm{MRC}}=\sum_{u=1}^{U} \gamma_{u}
$$

Therefore, $U$-orders diversity can be obtained in the scheme. However, it is strict that synchronization is required to ensure the precise estimation of the channel gain and phase distortion of each subchannel. In addition, transmission rate is reduced, because each symbol is repetitively transmitted in $U$ subchannels. Practically, to obtain only 2 -order diversity, the transmission efficiency will be halved. In addition, the average SNR should be equal in all subchannels in order to obtain optimum results from MRC that is completely difficult in some communication system.

2.3. Space-Frequency Block Code OFDM. In SFBC-OFDM both frequency and space are utilized to design the 2dimensional code words, which is used to obtain a diversity 
gain. Signal is distributed in the 2-dimensional space [13, 20]. Assume that the numbers of transmitter antennas and subchannels are, respectively, $M t$ and $N$; the SFBC code words are

$$
\mathbf{A}=\left[\begin{array}{cccc}
s_{1}(0) & s_{2}(0) & \cdots & s_{M t}(0) \\
s_{1}(1) & s_{2}(1) & \cdots & s_{M t}(1) \\
\vdots & \vdots & \ddots & \vdots \\
s_{1}(N-1) & s_{2}(N-1) & \cdots & s_{M t}(N-1)
\end{array}\right]
$$

where $s_{p}(n)$ is the symbol in the $n$th subchannel from the $p$ th transmit antenna $[6,13,14]$. After applying IFFT to the code words and adding cyclic prefix, symbols are transmitted through $M t$ antennas. SFBC requires that the coherence bandwidth should be at least twice the bandwidth of OFDM subchannel. In case of frequency selective channel, the coherence bandwidth may match the bandwidth of OFDM subchannel, resulting in mutual independent channels; consequently, SFBC will become functionless. To overcome the problem of obtaining full diversity when the subchannels of OFDM system are independent of each other, a pairwise code words joint SFBC scheme is proposed in [12]. The expression of the code words is

$$
\mathbf{C}=\left[\begin{array}{rrrrrrrr}
d_{1}^{1} & -d_{2}^{1^{*}} & d_{1}^{2} & -d_{2}^{2^{*}} & d_{3}^{1} & -d_{4}^{1^{*}} & d_{3}^{2} & -d_{4}^{2^{*}} \\
d_{2}^{1} & d_{1}^{1^{*}} & d_{2}^{2} & d_{1}^{2^{*}} & d_{4}^{1} & d_{3}^{1^{*}} & d_{4}^{2} & d_{3}^{2}
\end{array}\right] .
$$

Each row in (8) is transmitted through one antenna; thus $M t=2$ antennas are needed. $C$ consists of four Alamouti blocks, where $d_{i}^{1}(i=1, \ldots, 4)$ is the first data set and $d_{i}^{2}$ is the second data set. Since the transmitted symbols are joint designed, the joint detection is needed in the receiver. Consequently, $4 M r$-order diversity can be obtained when applying this scheme, where $M r$ is the number of receive antennas $[13,20]$.

Generally, SFBC is a diversity method that simultaneously uses space diversity and frequency diversity by using multiantennas and multisubchannels $[10,12,19]$. However, the use of multiantennas increases the practical implementation cost of the system.

\section{The Proposed Frequency Diversity}

3.1. Problem Statement and Analysis. Assume that there are $N$ independent subchannels and the ICI between them does not exist. Suppose that symbols belonging to different subchannels are transmitted independently and the complex output of the QAM/BPSK modulation is

$$
s_{j}=s_{I j}+i s_{\mathrm{Qj}},
$$

where $s_{j}$ is the modulation symbol from QAM/BPSK constellation of the $j$ th subchannel, $i=\sqrt{-1}$. The output vector of the QAM/PSK symbols is $\left[\mathbf{s}_{1}, \mathbf{s}_{2}, \ldots, \mathbf{s}_{\mathrm{N}}\right]^{T}$. The output of the IFFT of OFDM scheme is $s=\left[\begin{array}{llll}\dot{\mathbf{s}}_{1} & \dot{\mathbf{s}}_{2} & \cdots & \dot{\mathbf{s}}_{\mathrm{N}}\end{array}\right]^{\mathrm{T}}$. Suppose that the path gain and additive white Gaussian noise are $\alpha j$ and $W_{j}$, respectively. Accordingly, the received signal is a complex vector $r_{n}, n=1,2, \ldots, N$; the received signal can be expressed by

$$
\mathbf{r}_{\mathbf{n}}=\left(\mathbf{s}_{\mathrm{n}} * \boldsymbol{\alpha}_{\mathrm{n}}\right)+\mathbf{w}_{\mathbf{n}}
$$

In case $\alpha_{n}$ is deeply faded in such a way as no diversity advantages exist, the transmitted signal $s_{n}$ cannot recover without errors, because the symbols in the vector $\mathbf{s}$ are completely independent of each other. It is impossible for the receiver to make a correct decision on the received signal unless some forms of diversity are employed. Several diversity techniques, such as time, frequency, and spatial and code diversities, have been developed, to combat undesirable conditions of wireless channels $[1,2]$. Considering MRC-OFDM as one of these techniques, it can be mathematically modelled using the Relation Theory. We can say, MRC-OFDM uses two channels $f_{1}$ and $f_{2}$ to transmit two symbols $s_{1}$ and $s_{2}$. Although $s_{1}$ and $s_{2}$ are two different variables, $s_{1}$ practically is $s_{2}$ using the Equivalence Relation $\left(s_{1} \equiv s_{2}\right)$. That is to say, MRC transmits $M$ symbols using $M$ subchannels to obtain $M$-order diversity, but it simply applies the Equivalence Relation between these $M$ symbols. The transmitter side deals with these $M$ symbols as different $M$ symbols while the receiver, to take a decision, treats them as copies of one symbol. Hence, MRC consumes the transmitter resources needed for transmitting $M$ symbols, while these symbols are used together in the receiver side to detect only one symbol, because all these $M$ symbols are equivalent. Therefore, obtaining proper diversity, with minimum resources consumption and high diversity gain, mainly depends on the better selection of the relation between the symbols. This relation can be used by the receiver to detect a symbol and accordingly take a decision, while utilizing resources in both sides.

In mathematics Relation Theory, the relation between two elements $x, y$ or sets, can be the Equivalence Relation (三), a function $f(x, y)$, order relation, inverse relation, or many others. That way, sets of symbols can be ordered or there is an order relation that can be used to recognize them. The whole set can be classified, detected, or compared to some arbitrarily chosen element. Exploring such relations opens a wide research field that can be used to satisfy the requirements of particular communication systems.

In this paper, we will exploit the advantage of order theory and Hamming distance to overcome the diversity problem. Algebraic lattices are partially ordered sets such that a least upper bound and a greatest lower bound can be found for any subset consisting of two elements and [21] that means, for any two elements $x, y \in \mathbf{Q}, \operatorname{glb}\{x, y\}$ and $\operatorname{lub}\{x, y\}$ are exist. If $\mathbf{Q}$ set is a lattice then we can define $x \wedge y=\operatorname{glb}\{x, y\}$ and $x \vee y=\operatorname{lub}\{x, y\}[22,23]$. For the purpose of this paper the simple $M$ dimensional algebraic lattice is expressed by

$$
\mathbf{z}=\mathbf{G s},
$$

where $z$ is $M$ dimensional algebraic lattice. The components of vector $s=\left[\begin{array}{llll}s_{1} & s_{2} & \cdots & s_{M}\end{array}\right]^{T}$ are taken from the output of QAM/QPSK modulation; the symbols in $\mathbf{s}$ are independent of each other. $\mathbf{G}$ is $M \times M$ dimension matrix. $\mathbf{G}$ is known as the generator matrix [24]. The output vector 
$z=\left[\begin{array}{llll}z_{1} & z_{2} & \cdots & z_{M}\end{array}\right]^{T}$ is $M$ dimensional vector with some algebraic lattice characteristics. If the modulation order of the symbols in the signal vector $s$ is $P$, then there are $L=$ $P^{M}$ signal vectors. Let the $L$ vectors make up a vector set $\mathrm{Q}=\left[\begin{array}{llll}s_{1} & s_{2} & \cdots & s_{L}\end{array}\right]^{T}$. Accordingly, the corresponding lattice vector $z$ is of size $L$; then the set $Q^{\prime}=\left[\begin{array}{llll}z_{1} & z_{2} & \cdots & z_{L}\end{array}\right]^{T}$.

Motivated by error correction coding theory, Hamming code encodes $n$ data bits into $k$ bits by adding three parity bits. It can only detect and correct single-bit errors. It can also detect (but not correct) double-bit errors with the addition of an overall parity bit. Parity has a distance of 2, so one bit flip can be detected but not corrected and any two bit flips will be invisible. Hamming also noticed the problems with flipping two or more bits and described this as the distance. In general, the Hamming distance between two strings of equal length is the number of positions at which the corresponding symbols are different. In another way, it measures the minimum number of substitutions required to change one string into the other, or the minimum number of errors that could have transformed one string into the other. Suppose that $F$ is a finite field with $q$ elements. The Hamming distance $d(x, y)$ between two vectors $x, y \in F^{(n)}$ is the number of coefficients in which they differ, for example, in $F_{2}^{(5)} \rightarrow d(00111,11001)=4$, where $d$ satisfies the usual conditions for a metric $d(x, y) \geq 0$ and $d(x, y)=0$ if and only if $x=y$, because the number of coordinates in which $x$ differs from $y$ is equal to the number of coordinates in which $y$ differs from $x$. It also satisfies the metric $d(x, z) \leq d(x, y)+$ $d(y, z)$ for any $x, y, z \in F^{(n)}$, because $d(x, y)$ is equal to the minimal number of coordinate changes necessary to get from $x$ to $y$. In its turn, $d(y, z)$ is equal to the minimal number of coordinate changes necessary to get from $y$ to $z$. Therefore, $d(x, y)+d(y, z)$ changes will get us from $x$ to $z$. Hence, $d(x, y)+d(y, z) \geq d(x, z)$ which is the minimal number of coordinate changes necessary to get from $x$ to $z$. Generally, a certain code with distance $k$ can detect but not correct $(k-1)$ errors [25-27]. If more error-correcting bits are included with a message, and if those bits can be arranged such that different incorrect bits produce different error results, then bad bits could be identified. In a 7-bit message, there are seven possible single bit errors, so three error control bits could potentially specify not only that an error occurred but also which bit caused the error. Simply the $(n, k)$ code, can be modeled as $\mathbf{H G}^{\mathbf{T}}=\left(\mathbf{A} \mid \mathbf{I}_{n-k}\right) \times\left(\mathbf{I}_{\mathbf{k}} \mid \mathbf{A}^{T}\right)^{T}=0$, an all zero matrix for none block, where the matrix $\mathbf{G}$ here is also called the generator matrix of a linear $(n, k)$ code while $\mathbf{H}$ is called the parity check matrix, which is constructed by listing all columns of length $m=2,3, \ldots$ that are pairwise independent. Thus $\mathbf{H}$ is a matrix whose left part (A) is all of the nonzero $n$ tuples where order of the $n$-tuples in the columns of matrix does not matter. The matrix $\mathbf{I}_{n-k}$ is just the $(n-k)$-identity matrix. Therefore, $\mathbf{G}$ can be obtained from $\mathbf{H}$ by taking the transpose $\mathbf{A}$ with the identity $k$-identity matrix on the lefthand part of $\mathbf{G}$. These matrices can be mutated into equivalent nonsystematic codes by column permutations or elementary row operations $[25,27,28]$.
In general, the Hamming distance denotes the number of different code elements between two code words. For a certain code, the larger the minimum Hamming distance is the better the error correction performance is. In similar way, we define the number of different elements between two $M$ dimensional vectors as the vector Hamming distance. Obviously, as all the $M$ symbols in $\mathbf{s}$ are independent of each other, the minimum Hamming distance of the $L$ vectors in set $Q$ is typically 1 . However, by designing suitable generator matrix $\mathbf{G}$, algebraic lattice can achieve the minimum Hamming distance of vectors maximization in $Q^{\prime}$, that is, equal to the vector dimension $M$. Thus, symbols in the vector from $Q^{\prime}$ are interrelated with each other. Doing so dramatically mitigates the fading effects and greatly improves the antifading capability [24].

We utilized algebraic lattice theory to obtain frequency diversity gain, although it requires multimutual independent fades existing in the system. The good news is that OFDM can be used to transform frequency selective channel into multiple orthogonal subchannel in frequency domain and the fading in each subchannel is mutually independent. We exploited these characteristics accompanied with algebraic lattice and Hamming distance properties to design a new frequency diversity approach.

3.2. The Proposed Approach. The proposed OFDM diversity can improve the antifading capabilities of the OFDM system. The equivalent frequency domain of the proposed system is illustrated in Figure 2. We assume that there are $N$ independent OFDM subchannels. Then, we divide them into $p=$ $N / M$ sets; $N$ and $M$ usually are power of 2 . This means that there are $M$ subcarriers in each set and their corresponding fading is $\alpha_{1}^{k}, \alpha_{2}^{k}, \ldots, \alpha_{M}^{k}, k=1,2,3, \ldots, p$. Similarly, the modulated PSK/QAM symbols are also divided into $p$ sets, with $M$ symbols in each set. The symbols of each $p$ set are converted to $M$ dimensional algebraic lattice using (12). The output symbols $z=\left[z_{1}^{k}, z_{2}^{k}, \ldots, z_{M}^{k}\right]^{T}$ are transmitted over corresponding faded subchannels $\alpha_{1}^{k}, \alpha_{2}^{k}, \ldots, \alpha_{M}^{k}$; the received signal is $\mathbf{R}^{\prime}=(\mathbf{G S} * \boldsymbol{\alpha})+\mathbf{w}$. For the $k$ th set of $p$ and the symbols number $m$ in the same set, the received symbol can be expressed as (14), where $w_{m}^{k}$ is Gaussian white noise:

$$
\begin{gathered}
{\left[\begin{array}{c}
z_{1} \\
z_{2} \\
\vdots \\
z_{M}
\end{array}\right]^{T}=\mathbf{G}\left[\begin{array}{c}
s_{1} \\
s_{2} \\
\vdots \\
s_{M}
\end{array}\right]^{T},} \\
r_{m}^{k}=\alpha_{m}^{k} z_{m}^{k}+w_{m}^{k} .
\end{gathered}
$$

Suppose two or more different subchannels experience different fading. At least one of these subchannels does not fall into deep fading. Without losing generality, suppose $\alpha_{m}^{k}$ is the intended one. The receiver will detect the $m$ th dimension signal $z_{m}^{k}$, because using the basis of Hamming distance theory, $z_{m}^{k}$ is different from the $m$ th dimension signals of the rest $M-1$ vectors. Therefore, we can detect the transmission 


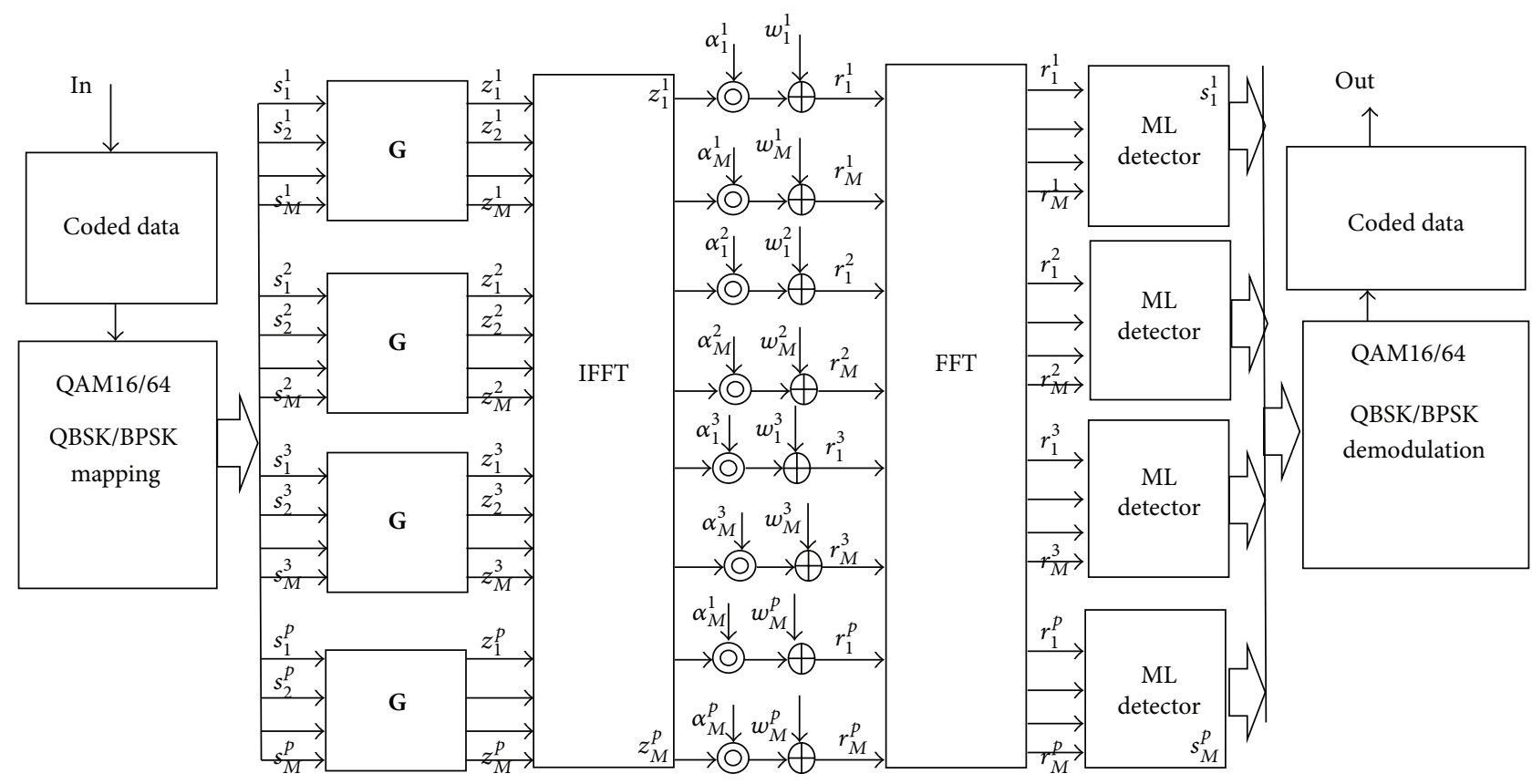

FIGURE 2: Frequency domain model of the proposed approach.

vector as $z^{k}$ just according to $z_{m}^{k}$. From the perspective of signal correlation, dimensions $z_{1}^{k}, z_{2}^{k}, \ldots, z_{M}^{k}$ of the $z^{k}$ are interrelated with each other due to the Hamming distance and algebraic lattice property. Motivated by information theoretic considerations, any signal in this vector contains the information of all the $M$ dimensional signals, because of the correlation. That is to say, the single symbol over a subchannel $\left(z_{m}^{k}\right)$ contains the entire information of the total vector. Hence, as long as $\propto_{1}^{k}, \propto_{2}^{k}, \ldots, \propto_{M}^{k}$ are not in deep fading at the same time, the receiver can recover the entire transmitted vector. When all $M$ subchannels are in deep fading, the receiver cannot recover the vector $z^{k}$. The probability that all subchannels are in deep fading is extremely low, particularly in case of presence of direct line of sight communication.

That means that the greater the amount of symbols in each set is the higher the diversity order is obtained; that is, the diversity order is equal to $M$. Moreover, since the symbols of each set are related to each other, their corresponding received symbols $r_{1}^{k}, r_{2}^{k}, \ldots, r_{M}^{k}$ will also be related to each other. Therefore, the receiver needs the joint detection. The maximum likelihood (ML) joint coding metric of the $M$ dimension lattice is according to

$$
\widehat{s}_{M \times 1}=\arg \min _{S_{M \times 1} \in s}\left\|r_{M \times 1}-\propto_{M \times M} \mathbf{z}_{M \times 1}\right\| .
$$

The design of $M$-order diversity system using the proposed approach mainly depends on three factors, which are the number of symbols $(M)$ in each set, the appropriate selection of the generator $G$, and the ML detector. This means that the complexity of the detector will increase corresponding to the $M$ value. Considering the compromise principle of the performance and complexity, we just choose a certain set size and make its diversity order meet some performance requirements. We design both the 2-order and 4-order system using the new proposed approach.

3.2.1. 2-Order Diversity. The 2-order diversity can be designed using the proposed approach by defining the corresponding lattice symbol generator and the ML detector. In this case $M=2, p=N / 2$. $M$ is the number of elements of each set p. $M$ also represents the number of symbols in each set. So, the symbols of each set can be represented by $s=\left[s_{1}, s_{2}\right]$. The input symbols $s_{1}, s_{2}$ are QAM/QPSK symbols. The generated lattice symbols $z=\left[z_{1}, z_{2}\right]$ can be obtained by applying (11), (12), and (15). The $G^{\prime}$ in this case is a rotation matrix according to [15]; however another type of generators is also acceptable:

$$
G^{\prime}=\left[\begin{array}{cc}
a & b \\
-b & a
\end{array}\right]
$$

where $a=1 / \sqrt{1+\rho^{2}}, b=a \rho$, and $\rho=(1 / 2)(1+\sqrt{5})$. The complex symbols are $s_{1}=s_{1 I}+j s_{1 \mathrm{Q}}$ and $s_{2}=s_{2 I}+j s_{2 \mathrm{Q}}$ in a similar way to that of (9). Then the real and imaginary parts are interleaved [29] and then rotated. The corresponding $z$ complex lattice symbols are $z_{1}=z_{1 I}+j z_{1 \mathrm{Q}}$ and $z_{2}=z_{2 I}+j z_{2 \mathrm{Q}}$, while the complete operation can be described by

$$
\left[\begin{array}{ll}
z_{1 I} & z_{1 \mathrm{Q}} \\
z_{2 I} & z_{2 \mathrm{Q}}
\end{array}\right]=G^{\prime}\left[\begin{array}{ll}
s_{1 I} & s_{1 \mathrm{Q}} \\
s_{2 I} & s_{2 \mathrm{Q}}
\end{array}\right] .
$$

As mentioned above, the subchannels of OFDM are independently faded. Therefore, each row of $z$ is transmitted over different subchannel. The frequency domain signal of 2dimensional lattice can be simplified using (17). The process 


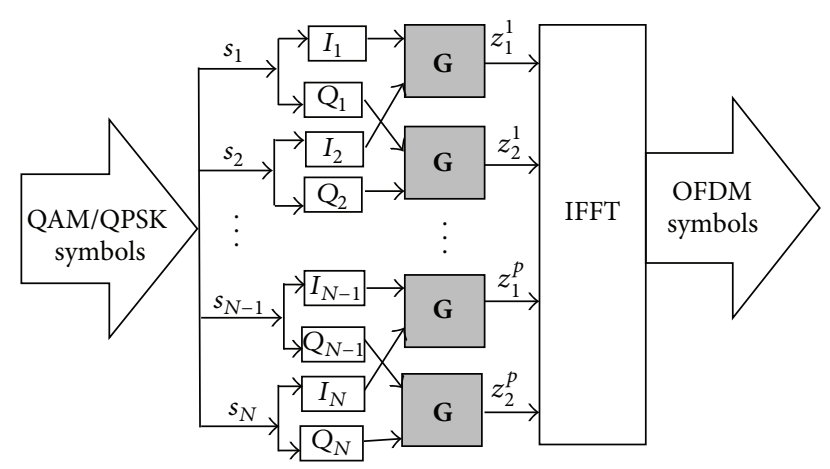

FIGURE 3: The process of obtaining 2-order frequency diversity using the proposed approach.

of obtaining 2-order frequency diversity using the proposed approach is simply described by Figure 3 :

$$
r_{2 \times 1}=\propto_{2 \times 2} z_{2 \times 1}+n_{2 \times 1} \text {. }
$$

In (17), $r_{2 \times 1}$ represents the received complex symbols, $z_{2 \times 1}=$ $G^{\prime} s_{2 \times 1}$, where the channel fading matrix $\propto_{2 \times 2}=\left[\begin{array}{cc}\alpha_{1} & 0 \\ 0 & \alpha_{2}\end{array}\right]$ and $n_{2 \times 1}$ is the 2-dimensional complex Gaussian white noise. Combining (11) and (16) into (14), we can obtain ML joint decoding metric of 2-dimensional lattice:

$$
\widehat{s}_{2 \times 1}=\arg \min _{s_{2 \times 1}}\left\|\left[\begin{array}{l}
r_{1} \\
r_{2}
\end{array}\right]-\propto_{2 \times 2}\left(G^{\prime}\left[\begin{array}{l}
s_{1 I} \\
s_{2 I}
\end{array}\right]+j G^{\prime}\left[\begin{array}{l}
s_{1 Q} \\
s_{2 Q}
\end{array}\right]\right)\right\| .
$$

3.2.2. Obtaining 4-Order Frequency Diversity. To achieve 4order frequency diversity, each set contains $M=4$ symbols. The input symbols are $s=\left[s_{1}, s_{2}, s_{3}, s_{4}\right]$; the generated lattice symbols are $z=\left[z_{1}, z_{2}, z_{3}, z_{4}\right]$. Many matrixes can function as a generator $G$, but we utilized the generator matrix defined by [30], which is a 4-dimensional complex lattice generator matrix:

$$
G_{4 \times 4}=\frac{1}{2}\left[\begin{array}{cccc}
\xi^{1 \times 1} & \xi^{2 \times 1} & \xi^{3 \times 1} & \xi^{4 \times 1} \\
\xi^{1 \times 5} & \xi^{2 \times 5} & \xi^{3 \times 5} & \xi^{4 \times 5} \\
\xi^{1 \times 9} & \xi^{2 \times 9} & \xi^{3 \times 9} & \xi^{4 \times 9} \\
\xi^{1 \times 13} & \xi^{2 \times 13} & \xi^{3 \times 13} & \xi^{4 \times 13}
\end{array}\right]
$$

where $\xi=e^{j 2 \pi / M}$; combining (19) into (11), we can achieve the 4-dimensional lattice transmitted vector as

$$
z_{4 \times 1}=G_{4 \times 4} s_{4 \times 1}
$$

In (20), $s_{1}, s_{2}, s_{3}$, and $s_{4}$ are symbols taken from PSK/QAM constellation. In similar way to that of (17), we can simply obtain the received vector as follows:

$$
r_{4 \times 1}=\propto_{4 \times 4} z_{4 \times 1}+n_{4 \times 1}
$$

where $r_{4 \times 1}$ is the received complex signal vector and the 4dimensional vector $\left(n_{4 \times 1}\right)$ is a complex Gaussian white noise:

$$
\propto_{4 \times 4}=\left[\begin{array}{cccc}
\propto_{1} & 0 & 0 & 0 \\
0 & \propto_{2} & 0 & 0 \\
0 & 0 & \propto_{3} & 0 \\
0 & 0 & 0 & \propto_{4}
\end{array}\right] .
$$

Equation (22) represents the channel fading matrix. Combining (19), (20), and (21) into (14), we can get the ML joint decoding metric of the 4-dimensional lattice ML detector as

$$
\widehat{s}_{4 \times 1}=\arg \min _{s_{4 \times 1}}\left\|r_{4 \times 1}-\propto_{4 \times 4} G_{4 \times 4} \mathbf{s}_{4 \times 1}\right\| .
$$

\section{Simulation Results and Observations}

We evaluated the new proposed system by implementing a simulation program using MATLAB. In the first part of the simulation, we examine the BER of the proposed approach and compare it with the traditional OFDM and MRC-OFDM diversity techniques. The OFDM symbols used in the simulation are transmitted over Rayleigh and AWGN channel, while forward error correction (FEC) mechanism is absent; however the presence of the FEC can improve the overall performance. In the simulation setup, we assume that the channel conditions are accurately estimated by the receiver, while a proper synchronization has been established. We used 64 subchannels; 48 are used for carrying randomly generated data symbols, 12 null, and 4 for pilots. All the subcarriers are in use throughout the simulations of both 2- and 4-order proposed systems. QAM16 is used as symbol baseband modulation to map the data as complex symbols in order to be modulated using IFFT. In MRC scheme, all the subcarriers are available, but the same QAM symbol is transmitted in 2 different subcarriers. The BER performance results of the first part of the simulation are shown in Figure 4.

Obviously, the use of the proposed approach utilizes all available subcarriers, unlike MRC-OFDM in which the symbol is sent using two subcarriers, which wastes the band width and consequently reduces the quality of communication (QoC). Out of $N$ subcarriers which include the data $\left(N_{u}\right)$, null subcarriers $\left(N_{L}\right)$, and pilot subcarriers $\left(N_{p}\right)$, only $N_{u} / M$ carriers are utilized. The actually utilized channels are equal to $\left(N-N_{p}-N_{L}\right) / M$. It can be observed from Figure 4 that the BER performance of the communication system has been significantly improved using diversity systems, while extra improvement is achieved using the proposed system. Above around $5 \mathrm{db}$, the system obtains the same performance of MRC-OFDM scheme using only the 2-order version of the proposed system, while it utilizes double of the available bandwidth. However, with the increase of SNR, the performance advantages of the 2-order proposed approach are more observable. Moreover, we also observed that the performance of the communication system is considerably improved using the 4-diversity version of the proposed approach. To obtain the same performance using MRC-OFDM diversity, we need to merge at least two MRC-OFDM systems that results in low bandwidth utilization. This means that we need $2 \times N$ 


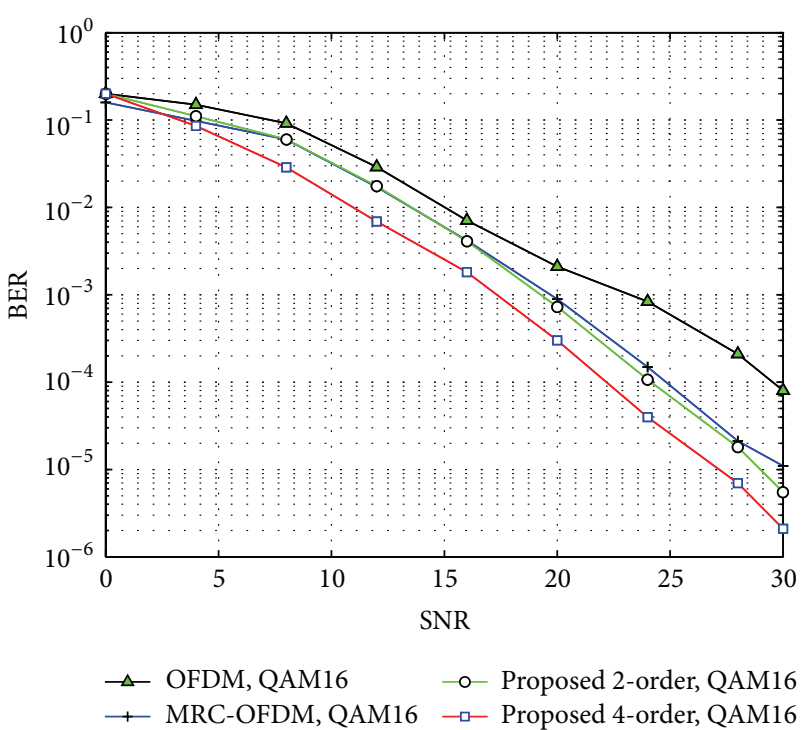

FIGURE 4: BER performance of the proposed approach compared to MRC-OFDM.

channels instead of $N$ to obtain the 4-order diversity, while reducing the amount of useful transmitted bits to $1 / 4$ of the total number of bits. This also means that the use of MRC reduces the useful transmitted bits to $N_{b} \times N / M$, where $N_{b}$ is the amount of bits per subcarrier and $M$ here is the diversity order. In contrast, the amount of useful bits using the proposed approach is $N_{b} \times N$. This amount is very important in many communication systems and applications.

From power consumption perspective, the useful bit energy spreads across $N_{u} / M$ subcarriers using MRC-OFDM, whereas the symbol energy spreads across $N$ subcarriers. The relationship between $E_{s}$ and $E_{b}$ is as below:

$$
\begin{gathered}
E_{s} \times N=E_{b} \times \frac{1}{M} N_{u}, \quad M=1,2, \ldots \\
\frac{E s}{N_{o}}=\frac{1}{M} \frac{N_{u}}{N} \frac{E_{b}}{N_{o}}, \quad \text { where } N_{o} \text { is white noise, } \\
\left(\frac{E_{s}}{N_{o}}\right)_{\mathrm{dB}}=\left(\frac{1}{M}\right)_{\mathrm{dB}}+\left(\frac{N_{u}}{N}\right)_{\mathrm{dB}}+\left(\frac{E_{b}}{N_{o}}\right)_{\mathrm{dB}} .
\end{gathered}
$$

This means that in each OFDM symbol $10 \log (1 / m) \mathrm{dB}+$ $10 \log \left(N_{u} / N\right) \mathrm{dB}$ is a wastage amount of bits energy, which allocated for MRC-OFDM diversity, null subcarriers, and pilot subcarriers. It is still interesting that the greatest amount of bits energy wastage $(10 \log (1 / M) \mathrm{dB})$ is due to MRCOFDM diversity. In contrast to that, the amount of power wastage in the proposed approach only depends on the number of pilot and null subcarriers.

To investigate the effect of the proposed diversity on the power of the OFDM symbol, we plot the power spectral density (PSD) of an OFDM symbol using (i) MRC-OFDM diversity with $N_{p}$ and $N_{L}$ subcarriers, (ii) MRC-OFDM diversity without $N_{p}$ and $N_{L}$, (iii) the proposed diversity approach with $N_{p}$ and $N_{L}$, and (iv) the proposed approach without $N_{p}$ and $N_{L}$. The PSD is normalized to the mean

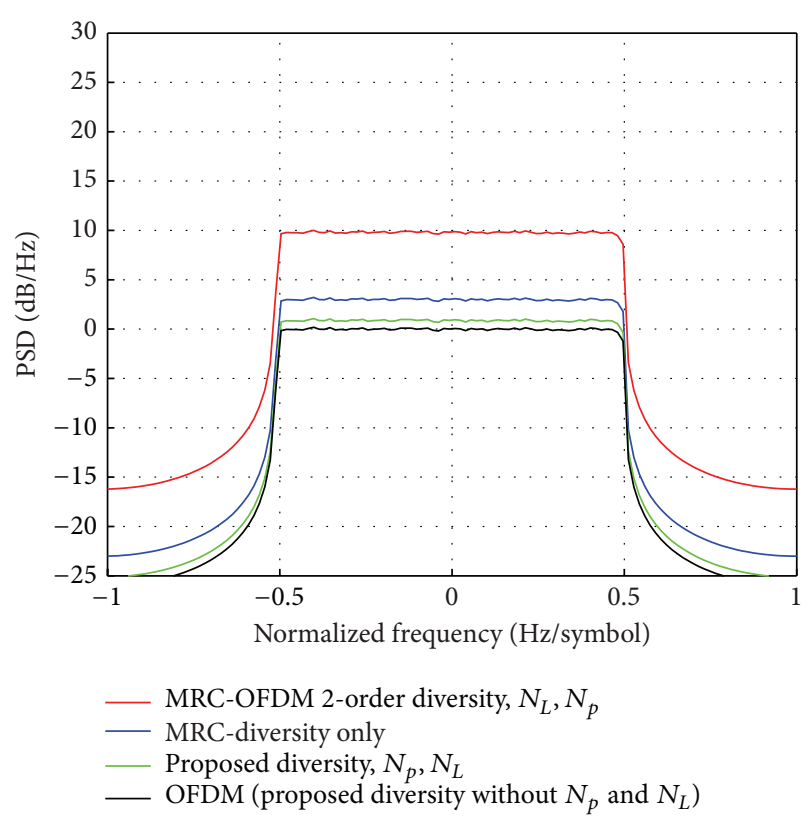

FIGURE 5: The effect of frequency diversity, pilot, and null on the power of an OFDM symbol.

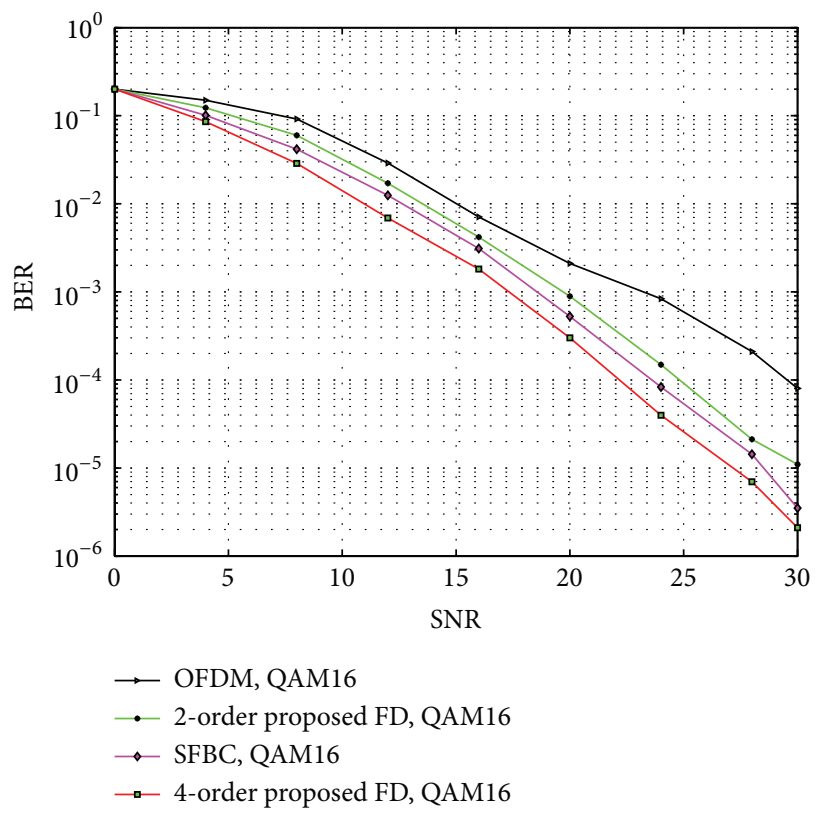

FIGURE 6: SNR versus BER of the proposed approach compared to SFBC and traditional OFDM diversity.

power of the data subcarriers. The result of this simulation is shown in Figure 5. It is obvious from Figure 5 that the wastage power due to MRC-OFDM diversity with $N_{p}$ and $N_{L}$ subcarriers is the highest one (around $10 \mathrm{db}$ is a wastage amount of power), while the proposed approach utilizes the energy of all useful bits in the OFDM symbol. 


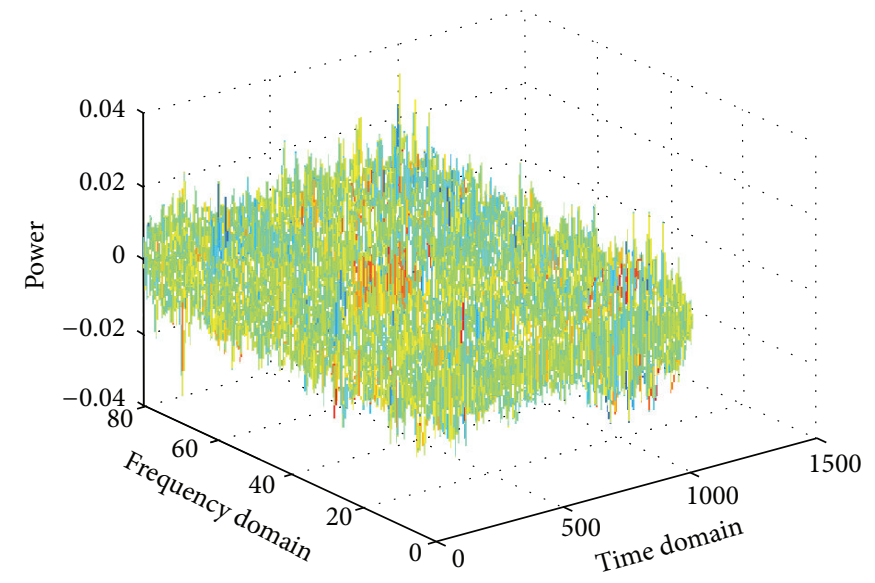

(a)

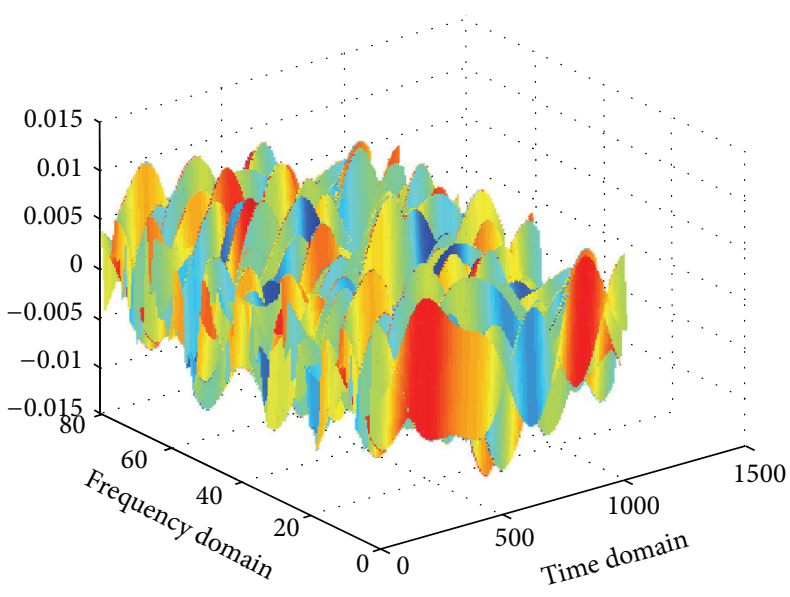

(b)

FIGURE 7: 3D representation of strongly faded signal (a) at the receiver input using traditional OFDM. (b) At the ML detector output using the 4-order diversity version of the proposed approach.

In the second part of the simulation, we compare the performance of the proposed 4-order diversity approach with the SFBC-OFDM [13]. We considered a SFBC-OFDM system with 2 transmit antennas and single receive antenna. In this simulation, Rayleigh channel is used with strong frequency selectivity; subchannels are considered independent of each other. We assume that the receiver has ideal channel estimation and synchronization. We use 64 subchannels and all the channels are in use with absence of channel coding techniques. Both the SFBC-OFDM that is in $[11,13]$ and the proposed approach are configured to use 16QAM modulation, with data rate equal to 256 bits per OFDM symbol (4 bits/subcarrier). It is obvious from Figure 6 that although both the proposed approach and SFBC-OFDM have 4-order diversity degree, the proposed approach has around $2.5 \mathrm{~dB}$ performance gain more than SFBC-OFDM.

Moreover, the proposed approach can transmit signals using only single-antenna system; nevertheless, SFBCOFDM scheme requires multiantenna system to obtain any order of diversity; consequently, the practical implementation is more complicated and that may also increase the implementation cost. Our proposed approach only needs the addition of the generator and the detector to the existing systems.

This does not mean that the use of multiple antenna systems with our proposed approach is not acceptable; on the contrary, the integration of MIMO system will add considerable improvement to the performance of the whole communication system, but the complicity and cost of the system may consequently increase. SFBC also requires that the coherence bandwidth should be at least twice the bandwidth of OFDM subchannel. This means that it is only suitable for certain situations and communication systems. It is also obvious from Figure 7 that the antifading capability of the new system is very high. Figure 7(a) shows the signals at the input of the receiver using traditional OFDM system, and Figure 7(b) shows the received signals after the ML detector.

\section{Conclusion}

In this paper, we presented a frequency diversity approach based on order theory, mainly algebraic lattice, to improve the OFDM system performance. Although the implementation of MRC-OFDM scheme is simple, it greatly reduces the transmission efficiency. On the other hand, SFBC-OFDM necessitates multiple antennas, suitable only for special kinds of communication systems, more complex, and costly. Compared with both MRC-OFDM and SFBC-OFDM, simulation results show that the proposed approach greatly gains performance improvement to the communication system and multiorder frequency diversity can be achieved by selecting the proper generator matrix and the suitable length of subset $(M)$. The use of the proposed approach only requires adding the symbols generator and the detection modules to the existing OFDM systems. It increases the communication performance without reduction in communication efficiency and with less practical implementation cost, while adding FEC to the system could close some of the remaining gaps. The new diversity approach can be integrated as a part of many communication systems that require high reliability, power consumption considerations, and high data rate. Moreover, the proposed system and SFBC-OFDM do not conflict with each other; in contrary, they can be integrated together for further improvement in QoC in some particular communication systems. Future work can concentrate on investigating other simple generators, in order to obtain high order diversity, in addition to the practical implementation issues of the proposed approach.

\section{Conflict of Interests}

The authors declare that there is no conflict of interests regarding the publication of this paper. 


\section{Acknowledgments}

This work is supported by National Natural Science Foundation of China under the Grant 61271204 and the National Key Technology R\&D Program under the Grant 2012BAH15B00.

\section{References}

[1] A. Bhandari, A. Kadambi, R. Whyte et al., "Resolving multipath interference in time-of-flight imaging via modulation frequency diversity and sparse regularization," Optics Letters, vol. 39, no. 6, pp. 1705-1708, 2014.

[2] H. Ali, A. Doucet, and Y. Hua, "Blind SOS subspace channel estimation and equalization techniques exploiting spatial diversity in OFDM systems," Digital Signal Processing, vol. 14, no. 2, pp. 171-202, 2004.

[3] C. Tepedelenlioglu, "Maximum multipath diversity with linear equalization in precoded OFDM systems," IEEE Transactions on Information Theory, vol. 50, no. 1, pp. 232-235, 2004.

[4] C. Gentile, N. Alsindi, R. Raulefs, and C. Teolis, "Multipath and NLOS mitigation algorithms," in Geolocation Techniques, pp. 59-97, Springer, New York, NY, USA, 2013.

[5] Y. Hui, B. Li, and Z. Tong, "The diversity gain and ICI suppression for OFDM systems over doubly selective channels," Wireless Personal Communications, vol. 77, no. 2, pp. 1465-1476, 2014.

[6] H. Bolcskei and A. J. Paulraj, "Space-frequency coded broadband OFDM systems," in Wireless Communications and Networking Conference (WCNC '00), pp. 1-6, 2000.

[7] T. Q. Wang, Y. A. Sekercioglu, and J. Armstrong, "Analysis of an optical wireless receiver using a hemispherical lens with application in MIMO visible light communications," Journal of Lightwave Technology, vol. 31, no. 11, Article ID 6497451, pp. 1744-1754, 2013.

[8] N. Chide, S. Deshmukh, and P. Borole, "Implementation of OFDM system using IFFT and FFT," International Journal of Engineering Research and Applications, vol. 3, pp. 2009-2014, 2013.

[9] K. N. Le and K. P. Dabke, "BER of OFDM with diversity and pulse shaping in Rayleigh fading environments," Digital Signal Processing, vol. 20, no. 6, pp. 1687-1696, 2010.

[10] M. Z. Win and J. H. Winters, "Analysis of hybrid selection/ maximal-ratio combining in Rayleigh fading," in Proceedings of the IEEE International Conference on Communications (ICC '99), pp. 6-10, 1999.

[11] S. Lu, B. Narasimhan, and N. Al-Dhahir, "A novel SFBC-OFDM scheme for doubly selective channels," IEEE Transactions on Vehicular Technology, vol. 58, no. 5, pp. 2573-2578, 2009.

[12] Z. Mohammadian, M. Shahabinejad, and S. Talebi, "New fulldiversity space-frequency block codes based on the OSTBCs," IEEE Communications Letters, vol. 16, no. 10, pp. 1620-1623, 2012.

[13] S. Yiu, D. Calin, O. Kaya, and K. Yang, "Distributed STBCOFDM and distributed SFBC-OFDM for frequency-selective and time-varying channels," in Proceedings of the Wireless Communications and Networking Conference (WCNC '12), pp. 251-255, IEEE, Shanghai, China, April 2012.

[14] J. Xiong, Q. Huang, Y. Xi, D. Ma, and J. Wei, "Multiple carrier frequency offsets tracking in co-operative space-frequency block-coded orthogonal frequency division multiplexing systems," IET Communications, vol. 7, no. 3, pp. 263-269, 2013.
[15] J. Boutros, E. Viterbo, C. Rastello, and J.-C. Belfiore, "Good lattice constellations for both Rayleigh fading and Gaussian channels," IEEE Transactions on Information Theory, vol. 42, no. 2, pp. 502-518, 1996.

[16] N. Prasad and M. K. Varanasi, "Analysis and optimization of diagonally layered lattice schemes for MIMO fading channels," IEEE Transactions on Information Theory, vol. 54, no. 3, pp. $1162-1185,2008$

[17] G. Pandey and A. Goel, "Long reach colorless WDM OFDMPON using direct detection OFDM transmission for downstream and OOK for upstream," Optical and Quantum Electronics, vol. 46, no. 12, pp. 1509-1518, 2014.

[18] V. Sharma and J. Singh, "On the BER evaluation for wireless OFDM system over multipath fading channels," Wireless Communication, vol. 5, pp. 114-118, 2013.

[19] A. F. Molisch, Wireless Communications, vol. 15, John Wiley \& Sons, New York, NY, USA, 2012.

[20] W. Zhang, X.-G. Xia, and K. Ben Letaief, "Space-time/frequency coding for MIMO-OFDM in next generation broadband wireless systems," IEEE Wireless Communications, vol. 14, no. 3, pp. 32-43, 2007.

[21] G. Birkhoff, Lattice Theory, vol. 25, American Mathematical Society, New York, NY, USA, 1948.

[22] X. Ma, W. Zhang, and A. Swami, "Lattice-reduction aided equalization for OFDM systems," IEEE Transactions on Wireless Communications, vol. 8, no. 4, pp. 1608-1613, 2009.

[23] S. Z. Budišin and P. Spasojević, "A generalized Boolean function generator for complementary sequences," in Proceedings of the IEEE Information Theory and Applications Workshop (ITA '14), pp. 1-7, February 2014.

[24] X. Giraud, E. Boutillon, and J.-C. Belfiore, "Algebraic tools to build modulation schemes for fading channels," IEEE Transactions on Information Theory, vol. 43, no. 3, pp. 938-952, 1997.

[25] A. Belal, B. Owaidat, N. Saleh, and R. Jaber, "Error correction capabilities in block ciphers," in Proceedings of the 2nd International Conference on Advances in Computational Tools for Engineering Applications (ACTEA '02), pp. 66-69, 2012.

[26] Y. Yang, "Information theory, inference, and learning algorithms," Journal of the American Statistical Association, vol. 100, no. 472, pp. 1461-1462, 2005.

[27] M. Norouzi, D. M. Blei, and R. Salakhutdinov, "Hamming distance metric learning," in Advances in Neural Information Processing Systems, pp. 1061-1069, 2012.

[28] T. K. Moon, Error Correction Coding: Mathematical Methods and Algorithms, John Wiley \& Sons, 2005.

[29] G. Taricco and E. Viterbo, "Performance of component interleaved signal sets for fading channels," Electronics Letters, vol. 32, no. 13, pp. 1170-1172, 1996.

[30] G. Wang, H. Liao, H. Wang, and X.-G. Xia, "Systematic and optimal cyclotomic lattices and diagonal space-time block code designs," IEEE Transactions on Information Theory, vol. 50, no. 12, pp. 3348-3360, 2004. 


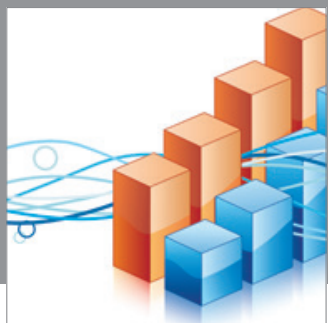

Advances in

Operations Research

mansans

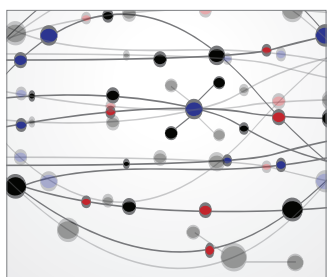

The Scientific World Journal
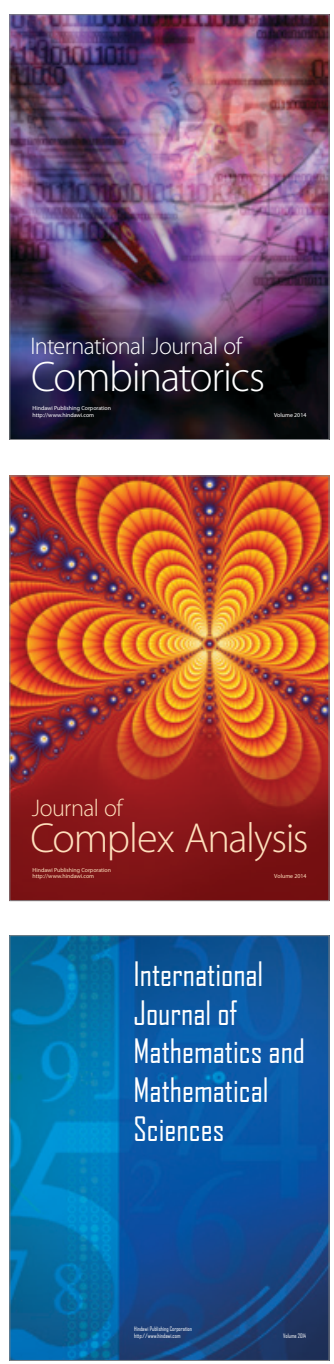
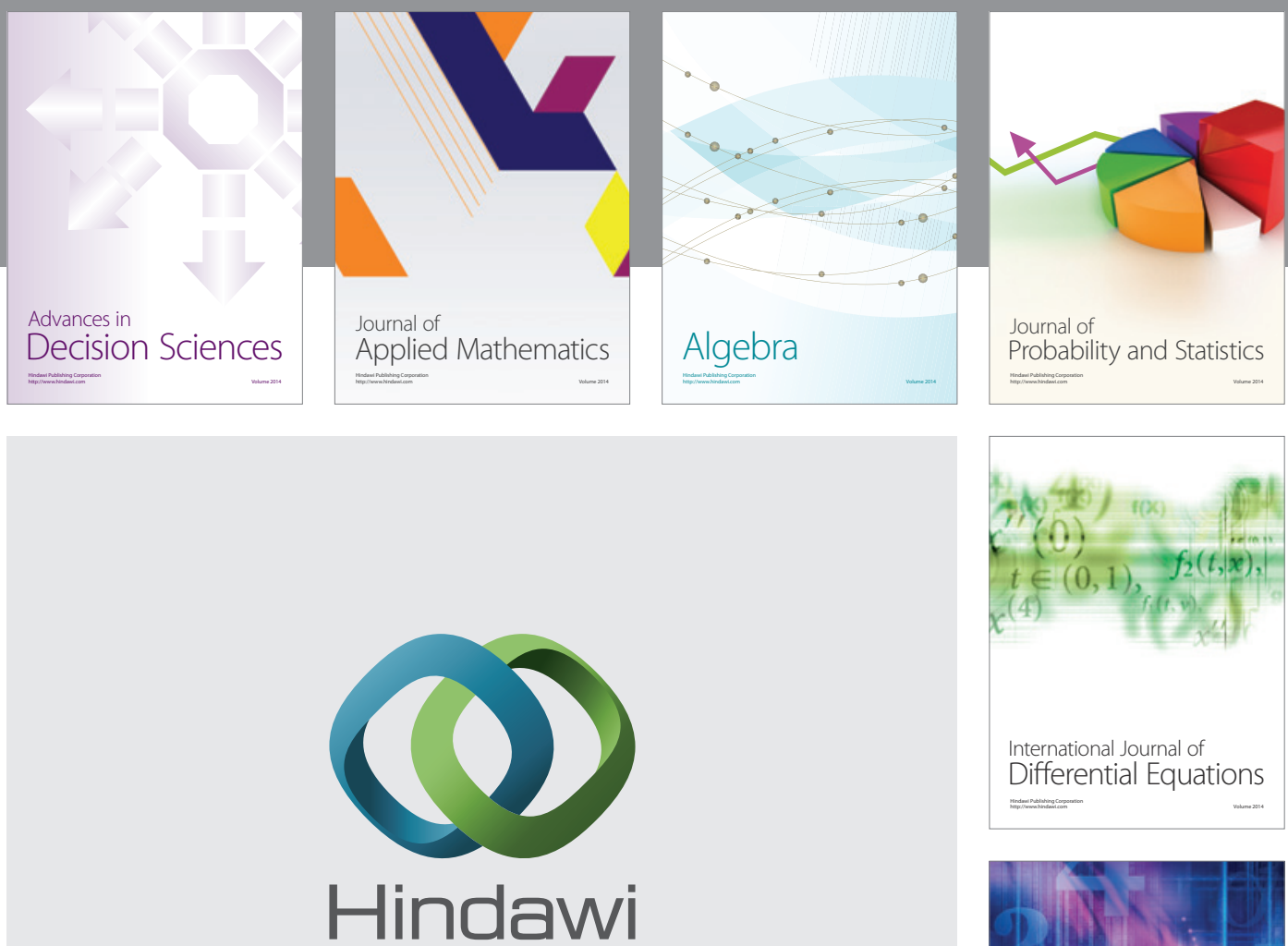

Submit your manuscripts at http://www.hindawi.com
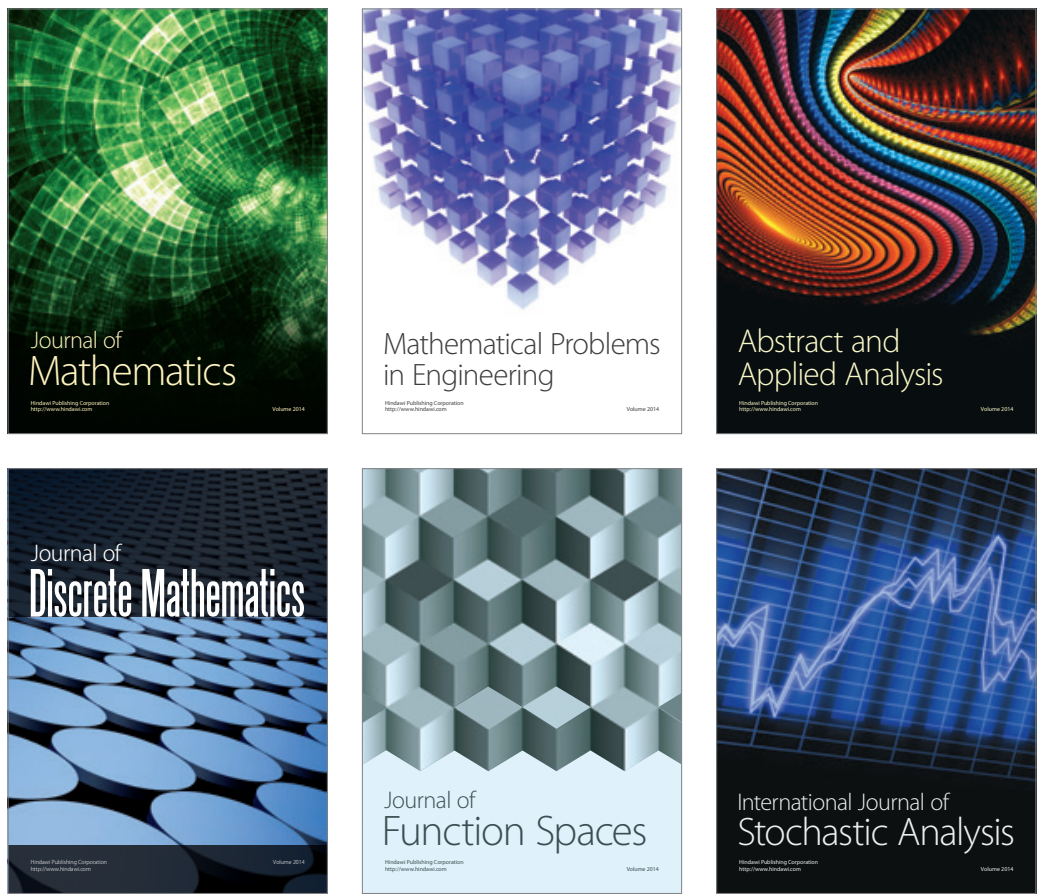

Journal of

Function Spaces

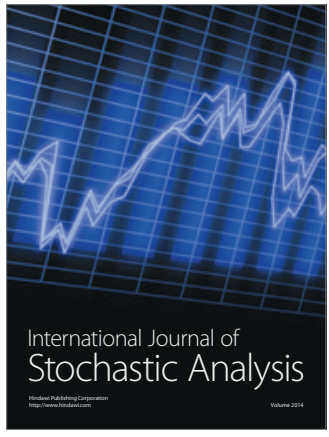

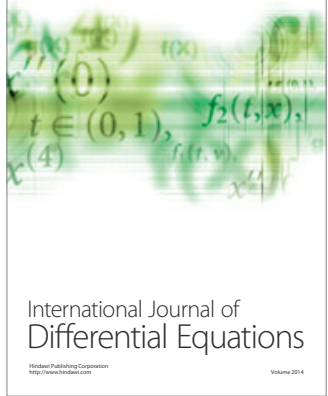
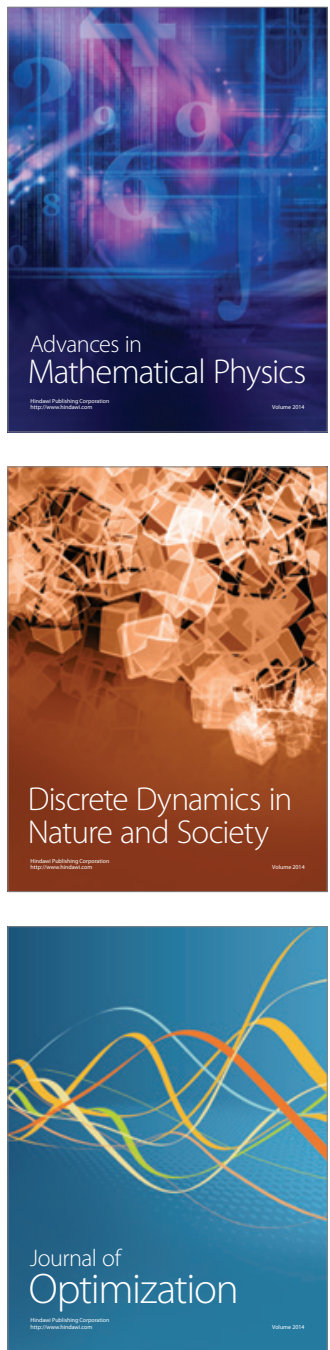